\title{
Successful Hemostasis Using an Over-the-scope Clip for a Dieulafoy's Lesion in the Greater Curvature of the Fundus
}

Dieulafoy's lesion is a relatively uncommon, occasionally fatal, cause of gastrointestinal bleeding. Endoscopy is useful for its diagnosis/treatment [1-3]. We report a case of bleeding from a Dieulafoy's lesion in the greater curvature of the fundus treated using an over-thescope clip (OTSC) (Ovesco Endoscopy AG, Tübingen, Germany).

A 72-year-old man presented with fainting and shock. Computed tomography revealed an exposed blood vessel with a major axis of $6 \mathrm{~mm}$ in the fundus and no extravasation ( $\mathbf{F i g . 1}$ ). Endoscopy revealed a Dieulafoy's lesion in the greater curvature of the fundus ( Fig.2). We performed endoscopic hemostasis using OTSC ( Video 1). The following day, hemostasis was confirmed on endoscopy ( Fig.3). The patient was discharged on hospital day 5 .

Dieulafoy's lesion in the stomach commonly occurs in the lesser curvature [2]. A lesion can be detected by endoscopy in only $71 \%$ of patients [1], because securing the visual field is difficult owing to the large amount of blood and small lesion size [2]. Moreover, as in this case, blood and food residue accumulate in the greater curvature of the fundus, making it difficult to locate a lesion during urgent endoscopy. Rebleeding during endoscopic treatment causes further difficulty in securing a visual field. For Dieulafoy's lesions, injection treatment, thermal coagulation, and mechanical therapy (clipping and band ligation) are endoscopic hemostasis approaches. Although outcomes have improved because of advances in endoscopy, results remain unsatisfactory owing to difficulty in hemostasis $(5.9 \%)$, rebleeding $(10.2 \%)$, transition to surgical treatment (4.6\%), and death (1.4\%) [3].

OTSC is effective as a first-line and second-line treatment for peptic ulcers $[4,5]$ (injection therapy and throughthe-scope clips are ineffective). OTSC
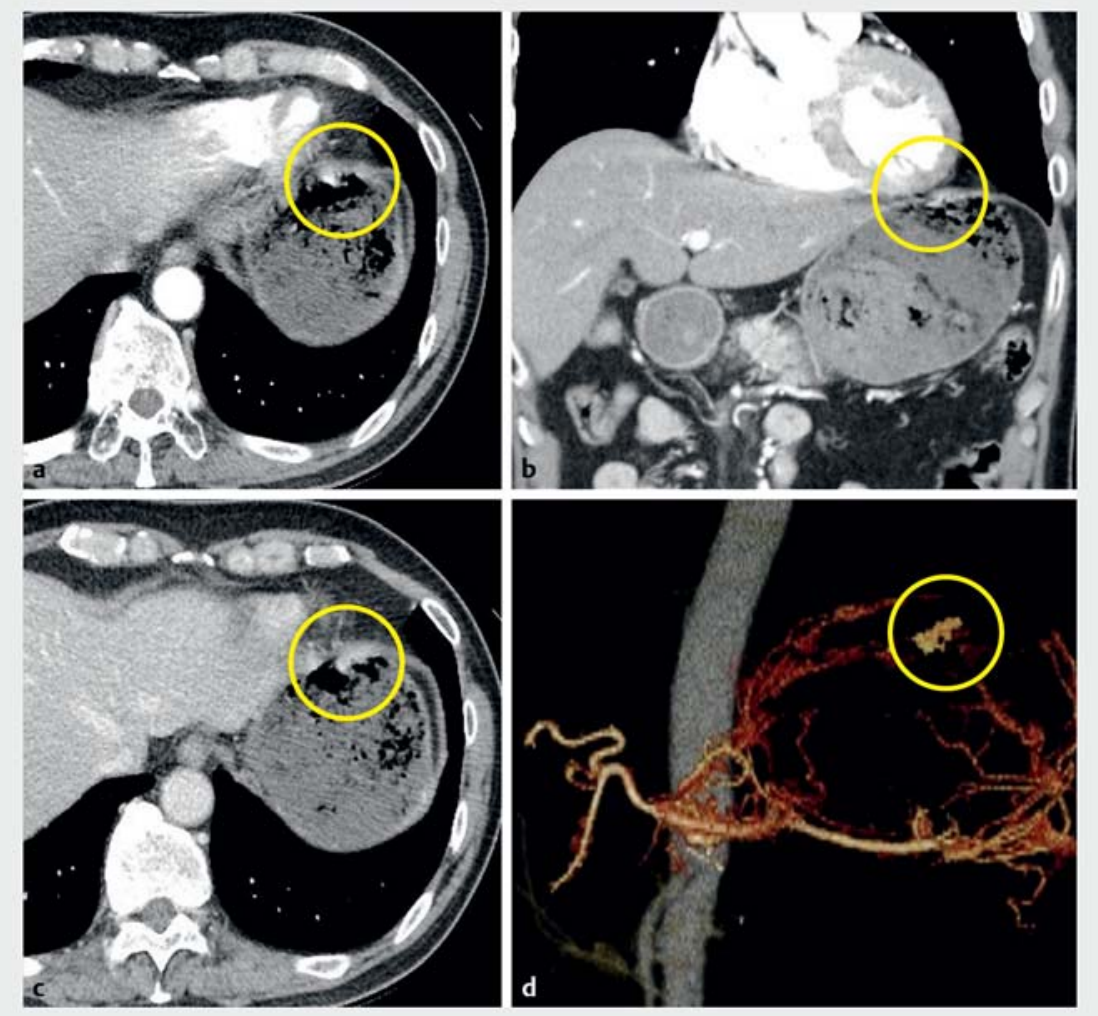

- Fig. $1 \mathrm{CT}$ image of an abnormally expanded, meandering submucosal artery with a major axis of $6 \mathrm{~mm}$ was observed in the fundus of the stomach in: a axial image of the arterial phase, and $\mathbf{b}$ coronal image of the arterial phase. There was no clear extravasation. c Axial image of the equilibrium phase. There was no clear extravasation in the equilibrium phase. $\mathbf{d}$ Threedimensional computed tomography angiography. There were abnormal expansion/meanderings of the left gastric artery in the fundus.

was selected based on the large vessel diameter and lesion site in this case, because we believe OTSC does not induce bleeding, unlike other mechanical hemostatic devices.

We considered OTSC to be an effective device for treating a Dieulafoy's lesion.

Endoscopy_UCTN_Code_TTT_1AO_2AD

Competing interests

None
The authors

Yo Kubota ${ }^{1}$, Hiroshi Yamauchi ${ }^{1,2}$, Kento Nakatani ${ }^{2}$, Satoshi Tanabe ${ }^{3}$, Wasaburo Koizumi ${ }^{1}$

1 Department of Gastroenterology, Kitasato University School of Medicine, Sagamihara city, Kanagawa, Japan

2 Department of Emergency and Disaster Medicine, Kitasato University School of Medicine, Sagamihara city, Kanagawa, Japan

3 Department of Research and Development Center for New Frontiers, Kitasato University School of Medicine, Sagamihara, Japan 

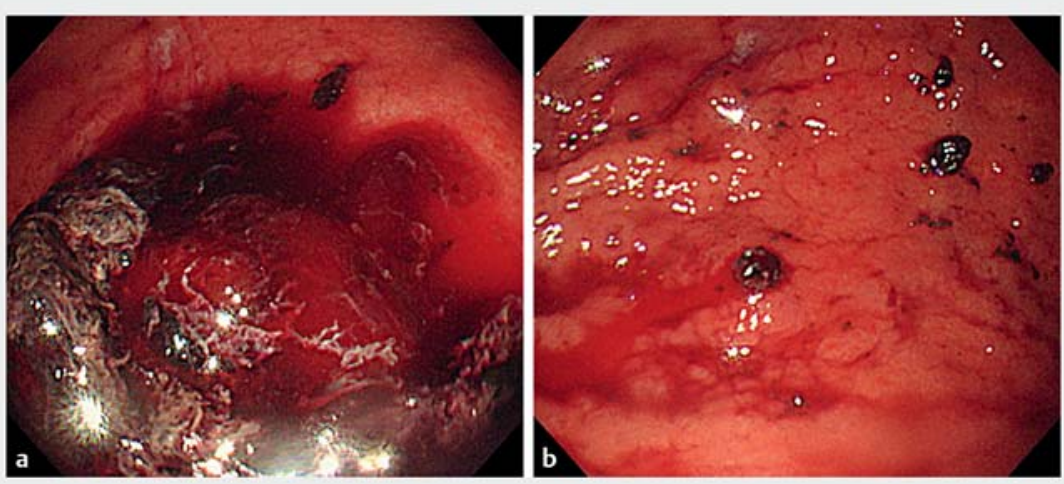

- Fig. 2 Urgent upper endoscopy. a There was a blood clot in the greater curvature of the fundus. Because there was no clear bleeding source other than the fundus, the blood clot in the fornix was removed by suction resulting in fresh bleeding. $\mathbf{b}$ Blood gushed from a Dieulafoy's lesion in the greater curvature of the fundus.
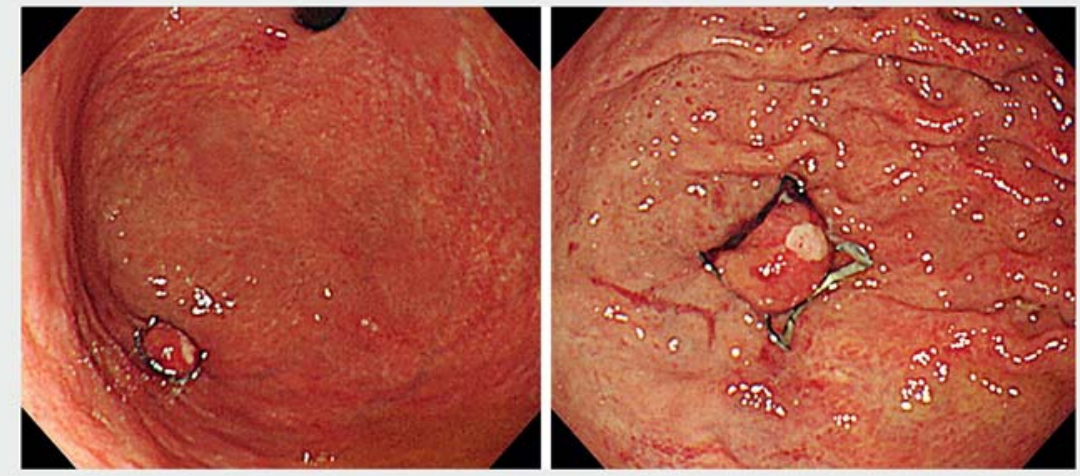

Fig. 3 Endoscopy on the following day. There was no clear bleeding, and the exposed blood vessel had been flattened.
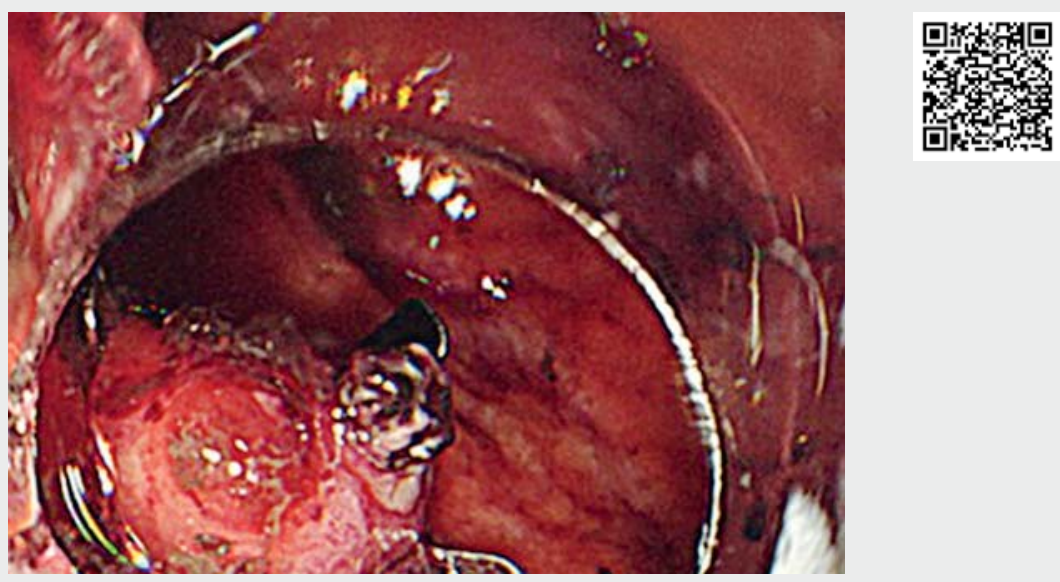

Video 1 Endoscopic hemostasis using an over-the-scope clip for a Dieulafoy's lesion in the greater curvature of the fundus.
Corresponding author

\section{Hiroshi Yamauchi, MD}

Department of Gastroenterology,

Department of Emergency and Disaster Medicine, Kitasato University School of

Medicine, 1-15-1 Kitasato, Minami-ku,

Sagamihara, Kanagawa, 252-0375 Japan

Fax: +81-42-778-8390

yhiroshi@kitasato-u.ac.jp

References

[1] Romaozinho JM, Pontes JM, Lerias C et al. Dieulafoy's lesion: management and longterm outcome. Endoscopy 2004; 36: 416420

[2] Baxter M. Dieulafoy's lesion: current trends in diagnosis and management. Ann R Coll Surg Engl 2010; 92: 548-554

[3] Jeon HK, Kim GH. Endoscopic management of Dieulafoy's lesion. Clinical Endosc 2015; 48: 112

[4] Manta R, Mangiafico S, Zullo A et al. Firstline endoscopic treatment with over-thescope clips in patients with either upper or lower gastrointestinal bleeding: a multicenter study. Endosc Int Open 2018; 6: E1317E1321

[5] Stefan G, Lukas N, Denis F et al. Over-thescope clip in peptic ulcer bleeding: clinical success in primary and secondary treatment and factors associated with treatment failure. Endosc Int Open 2019; 7: E846-E854

Bibliography

DOI https://doi.org/10.1055/a-1047-0174

Published online: 2.12.2019

Endoscopy 2020; 52: E164-E165

(c) Georg Thieme Verlag KG

Stuttgart · New York

ISSN 0013-726X

\section{ENDOSCOPY E-VIDEOS}

https://eref.thieme.de/e-videos

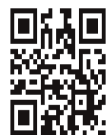

Endoscopy E-Videos is a free access online section, reporting on interesting cases and new techniques in gastroenterological endoscopy. All papers include a high quality video and all contributions are freely accessible online.

This section has its own submission website at

https://mc.manuscriptcentral.com/e-videos 